УДК 004.942

DOI: https://doi.org/10.17308/sait.2021.2/3503

ISSN 1995-5499

Поступила в редакцию 21.05.2021

Подписана в печать 19.07.2021

\title{
ИССЛЕДОВАНИЕ РЕШЕНИЯ ЗАДАЧИ ПАРАМЕТРИЧЕСКОЙ ИДЕНТИФИКАЦИИ МОДЕЛЕЙ РАСПРЕДЕЛЕННЫХ ДИНАМИЧЕСКИХ ПРОЦЕССОВ
}

\author{
(c) 2021 М. Г. Матвеев, Е. А. Сирота ${ }^{\bowtie}$ \\ Воронежский государственный университет \\ Университетская пл., 1, 394018 Воронеж, Российская Федераиия
}

\begin{abstract}
Аннотация. Идентификация параметров распределенных динамических систем является важной задачей технических, экономических и социальных приложений. При моделировании объектов таких приложений обычно используют уравнения многомерной авторегрессии с регрессорами, расположенными в смежных узлах пространственных координат. Очевидно, что в этом случае между регрессорами обычно существует значимая корреляционная зависимость. Возникает эффект квазимультиколлинеарности, следствием которого является завышенное значение стандартной ошибки оценок параметров авторегрессии, а также смещение получаемых оценок параметров. Среди различных методов повышения качества статистических оценок много таких, которые снижают стандартную ошибку и повышают смещение и, наоборот, среди них метод инструментальной переменной, ридж-регрессия и другие. Таким образом, наличие двух составляющих ошибки порождает необходимость поиска компромисса «смещения и разброса» хорошо известного в машинном обучении. Объектом нашего исследования являются уравнения множественной авторегрессии, полученные на основании аппроксимации однородных уравнений в частных производных с постоянными параметрами разностными уравнения со свойством консервативности. Разностная схема называется консервативной, если она сохраняет на сетке те же законы сохранения, что и в исходной дифференциальной задаче. В рамках данной работы проведен сравнительный анализ решения задачи параметрической идентификации в случае применения к ней обычного метода наименьших квадратов (МНК), ридж-регрессии и двух авторских методов понижения размерности. Сравнительный анализ применения исследуемых методов идентификации к оценке параметров показал существенную зависимость качества оценки от интенсивности помех наблюдения. При малых помехах все рассматриваемые методы успешно справляются с задачей идентификации. При увеличении интенсивности помех удовлетворительную работоспособность демонстрирует только авторский метод понижения размерности, основанный на учете свойства консервативности разностной схемы.

Ключевые слова: мультиколлинеарность, авторегрессионная модель, конечно-разностные уравнения, идентификация, МНК-оценки, смещенные оценок параметров модели, понижение размерности, ридж-регрессия.
\end{abstract}

\section{ВВЕДЕНИЕ}

Идентификация параметров распределенных динамических систем является одной из важнейших задач технических, экономических и социальных приложений. При моде-

Сирота Екатерина Александровна e-mail: sirota_ea@sc.vsu.ru лировании объектов этих приложений часто применяются пассивные методы идентификации, основанные на статистической обработке наблюдаемых значений переменных функционирующего объекта. При этом исходное линейное дифференциальное уравнение заменяется на соответствующую разностную схему $[1,2]$. Разностная схема позволяет перейти к регулярной сетке пространственных Контент доступен под лицензией Creative Commons Attribution 4.0 License. The content is available under Creative Commons Attribution 4.0 License. 
Исследование решения задачи параметрической идентификаиии моделей распределенных ...

и временных координат в узлах которой осуществляется наблюдение переменных. В этом случае для моделирования целесообразно использовать уравнения многомерной авторегрессии с регрессорами, расположенными в смежных узлах пространственных координат [3-5]. Очевидно, что в этом случае между регрессорами обычно существует значимая корреляционная зависимость. Возникает эффект квазимультиколлинеарности, следствием которого является завышенное значение стандартной ошибки оценок параметров авторегрессии [6]. Второй источник ошибок идентификации модели - корреляция случайных помех наблюдения переменных нестационарного процесса с регрессорами, следствием чего является смещение получаемых оценок параметров [6]. Среди различных методов повышения качества статистических оценок много таких, которые снижают стандартную ошибку и повышают смещение и наоборот. Например, уменьшение размерности признакового пространства авторегрессии, применение методов регуляризации снижает стандартную ошибку [13-15], но может увеличить смещение. Метод инструментальных переменных [15] эффективен при борьбе со смещением, но может увеличивать стандартную ошибку оценки.

Таким образом, наличие двух составляющих ошибки порождает необходимость поиска компромисса «смещения и разброса» хорошо известного в машинном обучении [13], частным случаем которого является рассматриваемая задача идентификации.

Объектом нашего исследования являются уравнения множественной авторегрессии, полученные на основании аппроксимации однородных уравнений в частных производных с постоянными параметрами разностными уравнения со свойством консервативности. Разностная схема называется консервативной, если она сохраняет на сетке те же законы сохранения, что и в исходной дифференциальной задаче [9].

Рассматривается приведенная разностная схема

$$
y_{i}^{k+1}=a_{1} y_{i-1}^{k}+a_{2} y_{i}^{k}+a_{3} y_{i+1}^{k},
$$

с заданными начальными и краевыми условиями:

$$
y_{i}^{0}=c_{i}, \quad y_{i-1}^{k}=b_{i-1}^{k}, \quad y_{i+1}^{k}=b_{i+1}^{k}, \quad \forall k,
$$

где $i$ - дискретные значения пространственной координаты, $k-$ дискретное время; $a_{1}+a_{2}+a_{3}=1$, что обеспечивает консервативность схемы [8]. Действительно, стационарный режим по всем координатам может обеспечиваться только когда правая часть выражения (1) представляет собой выпуклую линейную комбинацию.

Значения переменной $y_{i}^{k}$ измеряется в каждом узле $i$ с погрешностью $\xi_{i}^{k}$, формируемой случайным процессом типа «белый шум». Измеренное значение будем обозначать $x_{i}^{k}=y_{i}^{k}+\xi_{i}^{k}$. Тогда выражения (1) и (2) можно записать в форме авторегрессионной зависимости, описывающей, вообще говоря, нестационарный временной ряд:

$$
\begin{aligned}
& x_{i}^{k+1}=a_{1}\left(x_{i-1}^{k}-\xi_{i-1}^{k}\right)+a_{2}\left(x_{i}^{k}-\xi_{i}^{k}\right)+ \\
& +a_{3}\left(x_{i+1}^{k}-\xi_{i+1}^{k}\right)+\xi_{i}^{k+1}=a^{T} \cdot x^{k}+\omega_{i},
\end{aligned}
$$

где $\omega_{i}=\xi_{i}^{k+1}-a^{T} \cdot \xi^{k} ; T-$ здесь и далее знак транспонирования. Принципиальное отличие разностной схемы (1) от авторегрессии (3) состоит в том, что в правой части последней переменные $x_{i}^{k}, \forall i, k$ измеряются в узлах сетки. Значения начальных и краевых условий также определяются результатами измерений.

В рамках данной работы проведен сравнительный анализ решения задачи параметрической идентификации (3) в случае применения к ней обычного метода наименьших квадратов (МНК), ридж-регрессии и двух авторских методов понижения размерности $[10,11]$.

\section{1. МОДЕЛИ И МЕТОДЫ ИССЛЕДОВАНИЯ}

Оценки МНК параметров (3) имеют следующий вид [6]

$$
\hat{a}=\left(X^{T} X\right)^{-1} X^{T}(X a+\omega) .
$$

Наличие смещения проверяется применением оператора математического ожидания к выражению (4):

$$
a \neq a+\left(X^{T} X\right)^{-1} X^{T} \omega ;
$$


в условиях корреляции случайных помех наблюдения переменных нестационарного процесса с регрессорами. Следует отметить такое свойство оценок МНК как эффективность, т. е. минимальную дисперсию оценок параметров.

В качестве альтернативы МНК рассмотрим оценки параметров модели (3), получаемые с помощью ридж-регрессии. В этом случае к критерию МНК делается добавка регуляризации [14]

$$
Q_{p}(a)=\|x-y(a)\|^{2}+\tau\|a\|^{2} \rightarrow \min _{a},
$$

где $\tau-$ коэффициент регуляризации.

Регуляризованная МНК-оценка получается в виде

$$
\hat{a}_{p}=\left(X^{T} X+\tau I\right)^{-1} X^{T} y .
$$

У матриц $X^{T} X$ и $X^{T} X+\tau I$ собственные вектора совпадают, а собственные значения различаются на $\tau$. Поэтому число обусловленности для матрицы $X^{T} X+\tau I$ равно

$$
\mu\left(X^{T} X+\tau I\right)=\frac{\lambda_{\max }+\tau}{\lambda_{\min }+\tau} .
$$

Получается, что чем больше $\tau$, тем меньше число обусловленности. С ростом $\tau$ возрастает устойчивость задачи, но растет смещение оценок.

Нами были предложены методы понижения размерности за счет учета свойства консервативности разностной схемы $[10,11]$. Так в работе [10], с учетом коррелированности временных рядов в смежных узлах сетки, предложено уровень ряда в $i$-м узле заменить на выражение

$$
x_{i}^{k}=\beta_{1} x_{i-1}^{k}+\beta_{2} x_{i+1}^{k}+\xi^{k} .
$$

Выражение (7) позволяет рассчитывать на получение МНК-оценок $\hat{\beta}$ с меньшей стандартной ошибкой как за счет понижения размерности, так и вследствие снижения коррелированности временных рядов в несмежных узлах.

Подставляя выражение (7) в (3) получим

$$
\begin{aligned}
x_{i}^{k} & =\left(a_{1}+a_{2} \beta_{1}\right) x_{i-1}^{k}+\left(a_{2} \beta_{2}+a_{3}\right) x_{i+1}^{k}+ \\
& +\xi_{i}^{k+1}-\xi_{\Sigma}^{k}=\theta_{1} x_{i-1}^{k}+\theta_{2} x_{i+1}^{k}+\Delta \xi,
\end{aligned}
$$

где $\xi_{\Sigma}^{k}-$ выпуклая линейная комбинация по$\operatorname{Mex} \xi_{i-1}^{k}, \xi_{i}^{k}, \xi_{i+1}^{k}$.
Выражение (8) с полученными оценками $\hat{\beta}$ позволяет построить систему линейных уравнений относительно параметров $\hat{\beta}$ :

$$
\begin{aligned}
& a_{1}+a_{2} \hat{\beta}_{1}=\theta_{1}, \\
& a_{2} \hat{\beta}_{2}+a_{3}=\theta_{2}, \\
& a_{1}+a_{2}+a_{3}=1 .
\end{aligned}
$$

Определитель системы (9)

$$
\hat{\beta}_{1}+\hat{\beta}_{2}-1
$$

отличен от нуля в случае, когда

$$
\hat{\beta}_{1}+\hat{\beta}_{2} \neq 1 \text {. }
$$

Таким образом, оценки вычисляются в два этапа: сначала рассчитываются оценки $\hat{\beta}$ модели выражения (7), затем вычисляются оценки $\hat{\theta}$, и решается система (9).

Условие консервативности можно использовать напрямую [11]. Выражая, например, $a_{2}=1-a_{1}-a_{3}$ получим следующую модификацию модели выражения (3)

$$
\begin{gathered}
x_{i}^{k+1}-x_{i}^{k}= \\
=\hat{a}_{1}\left(x_{i-1}^{k}-x_{i}^{k}\right)+\hat{a}_{3}\left(x_{i+1}^{k}-x_{i}^{k}\right) .
\end{gathered}
$$

Здесь, также как и в предыдущем подходе, можно рассчитывать на получение МНК-оценок параметров с меньшей стандартной ошибкой.

Далее, приведенные четыре метода получения оценок, будем обозначать следующим образом: МНК - обычный МНК; РP ридж-регрессия; ДМНК- двухэтапный МНК; ММНК- модифицированный МНК.

\section{2. ЭКСПЕРИМЕНТАЛЬНОЕ ИССЛЕДОВАНИЕ КАЧЕСТВА ОЦЕНОК}

Исследование проводилось на примере одномерного по пространственной координате уравнения конвективной диффузии

$$
\begin{aligned}
& \frac{\partial y}{\partial \tau}=D \frac{\partial^{2} y}{\partial z^{2}}-v \frac{\partial y}{\partial z} \\
& y(0, z)=\varphi(z), \\
& y\left(t, z^{\min }\right)=f_{1}(t), \quad y\left(t, z^{\max }\right)=f_{2}(t) .
\end{aligned}
$$

где $D \geq 0$ - коэффициент диффузии, $v \geq 0-$ скорость конвекции, $z$ - пространственная координата.

Выборочная статистика, необходимая для модельного исследования была получена с 
Исследование решения задачи параметрической идентификаиии моделей распределенных ...

использованием аналитического решения уравнения (11) с заданными значениями параметров $D$ и $v$, начальными и граничными условиями.

Аналитическое решение задачи имеет вид [12]

$$
\begin{gathered}
x(t, l)=\exp \left(\frac{v}{2 D}\left(l-\frac{v t}{2}\right)\right)(\exp (-D t) \sin l+ \\
+\exp (-4 D t) \sin 2 l+\exp (-9 D t) \sin 3 l) .
\end{gathered}
$$

Рассматриваемое дифференциальное уравнение может быть представлено с помощью приведенной разностной схемы (1).

$$
\begin{aligned}
& y_{i}^{k+1}=a_{1} y_{i+1}^{k}+a_{2} y_{i}^{k}+a_{3} y_{i-1}^{k},\left(\frac{D \Delta t}{\Delta z^{2}}-\frac{v \Delta t}{2 \Delta z}\right)=0,2583 ; \\
& \text { где } a_{1}= \\
& a_{2}=\left(1-\frac{2 D \Delta t}{\Delta z^{2}}\right)=0,5 ; \\
& a_{3}=\left(\frac{D \Delta t}{\Delta z^{2}}+\frac{v \Delta t}{2 \Delta z}\right)=0,2417 .
\end{aligned}
$$

Нетрудно проверить, что $a_{1}+a_{2}+a_{3}=1$.

К значениям переменной $y_{i}$ в соответствующих узлах сетки была добавлена аддитивная помеха наблюдения $\xi_{i}^{k}$, полученная с помощью генератора независимых случайных чисел гауссовского типа с нулевым математическим ожиданием и единичной дисперсией $\sigma_{\xi}^{2}$. Интенсивность помехи $c \sigma_{\xi}^{2}$ задавалась на уровнях: $c=0.1, c=0.01, c=0.001$.

Для получения надежных результатов оценок смещения и стандартной ошибки, допускающих сравнения числовых величин, эксперименты во всех режимах повторялись 1000 раз и их результаты усреднялись.

Для каждого из четырех методов были получены оценки параметров исходной задачи идентификации, а также оценки средней величины смещения и стандартной ошибки. В случае ридж-регрессии рассматривались различные значения параметра регуляризации на каждом уровне шума.

В табл. 1 показано влияние значения параметра регуляризации ридж-регрессии на качество оценки параметра $a_{1}$ при интенсивности помех $c=0,01$. Для остальных параметров результаты качественно аналогичны.
Табл. 1 наглядно показывает, что с ростом параметра регуляризации стандартная ошибка оценки уменьшается, а смещение растет. Для сравнительного анализа ридж-регрессии с другими методами было выбрано значение параметра регуляризации $\tau=12,456$, при котором стандартная ошибка параметра $a_{1}$ соизмерима $\mathrm{c}$ соответствующим значением оценки МНК.

Таблица 1. Стандартная ошибка и смещение параметров в случае ридж-регрессии

[Table 1. Standard error and parameter offset in the case of ridge regression]

\begin{tabular}{|c|c|c|}
\hline $\begin{array}{c}\text { Параметр } \\
\text { регуляризации }\end{array}$ & $\begin{array}{c}\text { Стандартная } \\
\text { ошибка }\end{array}$ & Смещение \\
\hline 2.0498 & 0.09207 & 0.129 \\
\hline 4.4976 & 0.09201 & 0.138 \\
\hline 8.9851 & 0.0909 & 0.145 \\
\hline 12.456 & 0.09 & 0.152 \\
\hline 14.876 & 0.0806 & 0.201 \\
\hline 16.976 & 0.08 & 0.399 \\
\hline
\end{tabular}

В табл. 2 приведены результаты реализации методов МНК, РР, ДМНК и ММНК: оценки величины стандартной ошибки (СО) и смещения (CM) при идентификации параметров разностной схемы (1) с различной интенсивностью помех $c$.

Данные табл. 2 показывают, что при малых помехах $(c=0,001)$ все методы идентификации обеспечивают удовлетворительную оценку параметров разностной схемы (1) ошибка не более $1 \%$ от абсолютного значения параметра. При больших $(c=0,1)$ и средних $(c=0,01)$ помехах с идентификацией более или менее справляется только ММНК, обеспечивая стандартную ошибку и смещение оценок до 10 \% при больших помехах и до $4 \%$ при средних помехах. Другие методы демонстрируют практически не приемлемые значения стандартной ошибки и смещения.

При среднем уровне шума стандартная ошибка оценки методом РР несколько ниже соответствующей оценки МНК, что подтверждает теоретические ожидания. Стандартную ошибку можно было бы уменьшить, применяя PP, но как показано в табл. 1, при этом достаточно быстро начинает расти смещение. 


\section{М. Г. Матвеев, Е. A. Сирота}

Таблица 2. Стандартная очибка и смещение параметров в случае МНК, РР, ДМНК, ММНК

[Table 2. Standard error and parameter offset in the case of OLS, ridge regression, two-stage OLS, modified OLS]

\begin{tabular}{|c|c|c|c|c|c|}
\hline Параметры & $\mathrm{C}$ & MHK & $\mathrm{PP}$ & ДМНК & MMHK \\
\hline \multicolumn{6}{|c|}{ Средняя величина смещения } \\
\hline \multirow[t]{3}{*}{$\mathrm{a} 1=0,2583$} & 0,1 & 0,1375 & 0,678 & 0,1321 & 0,0300 \\
\hline & 0,01 & 0,0937 & 0,152 & 0,0900 & 0,0110 \\
\hline & 0,001 & 0,0030 & 0,0357 & 0,0010 & 0,0009 \\
\hline \multirow[t]{3}{*}{$\mathrm{a} 2=0,5$} & 0,1 & 0,1707 & 0,5680 & 0,1690 & 0,0286 \\
\hline & 0,01 & 0,1625 & 0,1986 & 0,1601 & 0,0160 \\
\hline & 0,001 & 0,0049 & 0,0456 & 0,0008 & 0,0004 \\
\hline \multirow[t]{3}{*}{$\mathrm{a} 3=0,2417$} & 0,1 & 0,0244 & 0,6450 & 0,0369 & 0,0020 \\
\hline & 0,01 & 0,0683 & 0,1745 & 0,0701 & 0,0010 \\
\hline & 0,001 & $-0,0017$ & $-0,0299$ & 0,0002 & 0,0001 \\
\hline \multicolumn{6}{|c|}{ Средняя величина стандартного отклонения } \\
\hline \multirow[t]{3}{*}{$\mathrm{a} 1=0,2583$} & 0,1 & 0,1351 & 0,1532 & 0,1320 & 0,0270 \\
\hline & 0,01 & 0,0922 & 0,0900 & 0,0900 & 0,0100 \\
\hline & 0,001 & 0,0021 & 0,0091 & 0,0009 & 0,0008 \\
\hline \multirow[t]{3}{*}{$\mathrm{a} 2=0,5$} & 0,1 & 0,1699 & 0,1622 & 0,1689 & 0,0271 \\
\hline & 0,01 & 0,1608 & 0,1501 & 0,1600 & 0,0156 \\
\hline & 0,001 & 0,0024 & 0,0020 & 0,0008 & 0,0004 \\
\hline \multirow[t]{3}{*}{$\mathrm{a} 3=0,2417$} & 0,1 & 0,0207 & 0,0201 & 0,0360 & 0,0019 \\
\hline & 0,01 & 0,0665 & 0,0602 & 0,0643 & 0,0008 \\
\hline & 0,001 & 0,0001 & 0,0001 & 0,0001 & 0,0001 \\
\hline
\end{tabular}

Наглядное представление результатов сравнительного анализа точности получения оценок параметров $a_{1} ; a_{2} ; a_{3}$ разностной схемы (1) для средней интенсивности помех наблюдения показаны на рис. 1 и 2.

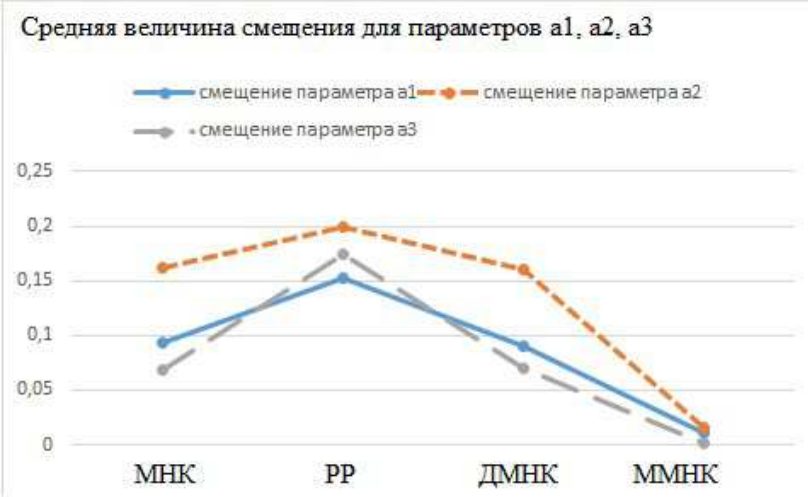

Рис. 1. Средняя величина смещения оченок параметров

[Fig. 1. The average value of the bias of the parameter estimates]
Средняя величина стандартной ошибки для параметров $\mathrm{a} 1, \mathrm{a} 2$, a3

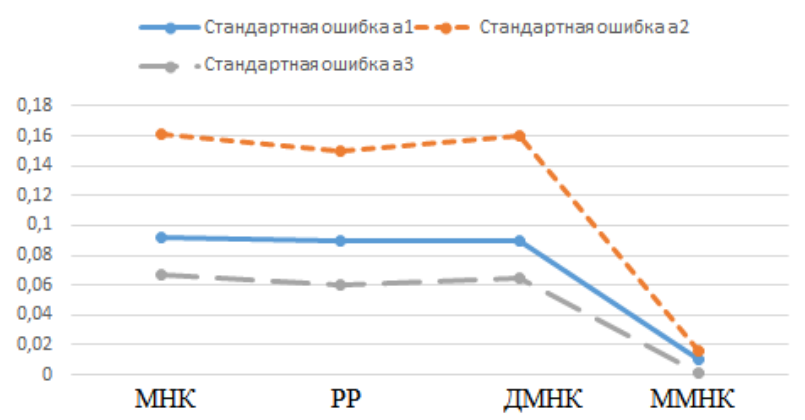

Рис. 2. Средняя величина стандартной ошибки оценок параметров

[Fig. 2. The average value of the standard error of parameter estimates] 
Исследование решения задачи параметрической идентификаиии моделей распределенных ...

\section{ЗАКЛЮЧЕНИЕ}

Сравнительный анализ применения исследуемых методов идентификации к оценке параметров консервативной разностной схемы (1) показал существенную зависимость качества оценки от интенсивности помех наблюдения. При малых помехах $(c=0,001)$ все рассматриваемые методы успешно справляются с задачей идентификации. При увеличении интенсивности помех $(c=0,01$ и $c=0,1)$ удовлетворительную работоспособность демонстрирует только модифицированный метод наименьших квадратов, основанный на учете свойства консервативности разностной схемы. Таким образом, модифицированный МНК можно рекомендовать для идентификации моделей распределенных динамических систем, разностное представление которых удовлетворяет приведенному свойству консервативности $a_{1}+a_{2}+a_{3}=1$. Следует ожидать, что в задачах с более высокой размерностью пространственных координат - $\left(R^{2}\right.$ и $\left.R^{3}\right)$, использование свойства консервативности также будет давать положительный эффект.

\section{КОНФЛИКТ ИНТЕРЕСОВ}

Авторы декларируют отсутствие явных и потенциальных конфликтов интересов, связанных с публикацией настоящей статьи.

\section{СПИСОК ЛИТЕРАТУРЫ}

1. Егориин, А. О. Кусочно-линейная идентификация и дифференциальная аппроксимация на равномерной решетке. / А. О. Егоршин. - Материалы XII Всероссийского совещания по проблемам управления. - М., 2014. - C. 2807-2822.

2. Безручко, Б. П. Современные проблемы моделирования по временным рядам / Б. П. Безручко, Д. А. Смирнов // Известия Саратовского университета, серия «Физика». 2006. - T. 6, № 1-2. - С. 3-27.

3. Guo, L. Z. Identification of partial differential equation models for a class of multiscale spatio-temporal dynamical systems / L. Z. Guo,
S. A. Billings, D. Coca // International Journal of Control. - 2010. - № 83:1. - P. 40-48. DOI: $10.1080 / 00207170903085597$

4. Матвеев, М. Г. Комбинированная прогностическая модель нестационарного многомерного временного ряда для построения пространственного профиля атмосферной температуры / М. Г. Матвеев, В. В. Михайлов, Е. А. Сирота // Информационные технологии. - 2016. - Т. 22, № 2. - С. 89-94.

5. Xiaolei, X. Parameter Estimation of Partial Differential Equation Models / Xun Xiaolei, Cao Jiguo, Mallick Bani, J. Carroll Raymond, Maity Arnab // Journal of the American Statistical Association. - 2013. -No 108:503. - P. 10091020, DOI: $10.1080 / 01621459.2013 .794730$

6. Носко, В. П. Эконометрика. Введение в регрессионный анализ временных рядов / В. П. Носко. - М. : НФПК, 2002. - 273 с.

7. Gareth, J. An Introduction to Statistical Learning / J. Gareth, D. Witten, Tr. Hastie, R. Tibshirani // Springer. - 2013. - P. 40-48.

8. Matveev, M. G. Modeling of Nonstationary Distributed Processes on the Basis of Multidimensional Time Series / M. G. Matveev, A. V. Kopytin, E. A. Sirota, E. A. Kopytina // Procedia Engineering. - 2017. - № 201. - P. 1-862. DOI: $10.1016 /$ j.proeng.2017.09.643

9. Самарский, А. А. Теория разностных схем / А. А. Самарский. - М. : Наука, 1978. $376 \mathrm{c}$.

10. Матвеев, М. Г. Анализ свойств МНК-оценок в случае устранения мультиколлинеарности в задачах параметрической идентификации распределенных динамических процессов / М. Г. Матвеев, Е. А. Сирота // Вестник Воронеж. гос. ун-та. Сер. Системный анализ и информационные технологии. - 2020. - № 2. C. $15-22$.

11. Matveev, M. G. Analysis and investigation of the conservativeness condition in the problem of parametric identification of distributed dynamic processes / M. G. Matveev, E. A. Sirota // Journal of Physics: Conference Series. - 2021. - № 1902. 012079. DOI:10.1088/1742-6596/1902/1/012079

12. Копьтин, А. В. Применение расширенного фильтра Калмана для идентификации параметров распределенной динамической системы / А. В. Копытин, Е. А. Копытина, 
М. Г. Матвеев // Вестник Воронеж. гос. ун-та. Сер. Системный анализ и информационные технологии. - 2018. - № 3. - С. 44-50.

13. Барсегян, A. A. Анализ данных и процессов: учеб. пособие / А. А. Барсегян, М. С. Куприянов, И. И. Холод, М. Д. Тесс, С. И. Елизаров. - 3-е изд., перераб. и доп. СПб. : БХВ-Петербург, 2009. - 512 с.

14. Tibshirani, R. J. Regression shrinkage and selection via the lasso / R. J. Tibshirani //
Journal of the Royal Statistical Society. Series B (Methodological). - 1996. - Vol. 58, No. 1. P. 267-288.

15. Айвазян, С. А. Прикладная статистика. Классификация и снижение размерности / С. А. Айвазян, В. М. Бухштабер, И. С. Енюков, Л. Д. Мешалкин. -М. : Финансы и статистика, 1989. -607 c.

Матвеев Михаил Григорьевич - д-р тех. наук, проф., заведующий кафедрой информационных технологий управления факультета компьютерных наук Воронежского государственного университета.

E-mail: mgmatveev@yandex.ru

ORCID iD: https://orcid.org/0000-0002-6528-6420

Сирота Екатерина Александровна - канд. физ.-мат. наук, доцент кафедры цифровых технологий факультета компьютерных наук Воронежского государственного университета.

E-mail: sirota_ea@sc.vsu.ru

ORCID iD: https://orcid.org/0000-0002-0338-7461

DOI: https://doi.org/10.17308/sait.2021.2/3503

Received 21.05.2021

Accepted 19.07.2021

ISSN 1995-5499

\title{
STUDYING THE SOLUTIONS TO THE PARAMETRIC IDENTIFICATION OF THE MODELS OF DISTRIBUTED DYNAMIC PROCESSES
}

\author{
(C) 2021 M. G. Matveev, E. A. Sirota \\ Voronezh State University \\ 1, Universitetskaya Square, 394018 Voronezh, Russian Federation
}

\begin{abstract}
Annotation. Identifying the parameters of dynamic distributed systems is an important task which has technical, economic, and social applications. When modelling the objects of such applications, multidimensional autoregression equations are normally used with regressors located at adjacent nodes of spatial coordinates. Obviously, in this case there is usually a significant correlation between the regressors. Here, we observe a quasimulticollinearity effect which results in an overestimated value of the standard error of the estimation of the autoregression parameters and biased estimates of the model's parameters. Methods of improving the quality of statistical estimates include a large number of those that reduce the standard error and increase the bias, and vice versa, such as the method of instrumental variables, ridge regression, etc. Thus, the presence of two components of error results in the need to find a compromise between bias and variance, a dilemma well-known in machine learning. In our study we focused on the multiple autoregression equations obtained by means of approximation of homogeneous partial differential equations with constant parameters to the difference equations with the property of conservativeness. A difference scheme is considered conservative if it preserves the same conservation laws on the

Sirota Ekaterina A.

e-mail: sirota_ea@sc.vsu.ru grid as in the original differential problem. The article presents the results of a comparative anal-
\end{abstract}


ysis of the following solutions to the problem of parametric identification: the ordinary least squares (OLS) method, ridge regression, and two methods of reducing the dimension suggested by the authors. The comparative analysis of the application of the studied identification methods to the estimation of the parameters showed a significant dependence of the quality of the assessment on the intensity of the observation interference. With low interference, all the considered methods are effective. With an increase in the intensity of interference, only the author's method of reducing the dimension taking into account the conservativeness of the difference scheme provides satisfactory results.

Keywords: autoregressive model, finite difference equations, identification, OLS estimates, biased estimates of model parameters, dimensionality reduction.

\section{CONFLICT OF INTEREST}

The authors declare the absence of obvious and potential conflicts of interest related to the publication of this article.

\section{REFERENCES}

1. Egorshin A. O. (2014) Piecewise-linear identification and differential approximation on a uniform grid. Proceedings of the XII all-Russian conference on problems of management. Moscow. P. 2807-2822.

2. Bezruchko B. P., Smirnov D. A. (2006) Modern problems of modeling from time series. Proceedings of the Sa-Raevskogo University, series "Physics". Vol. 6, No. 1-2. P. 3-27.

3. Guo L. Z., Billings S. A., Coca D. (2010) Identification of partial differential equation models for a class of mul-tiscale spatio-temporal dynamical systems. International Journal of Control, No 83:1. P. 40-48. DOI: 10.1080/00207170903085597

4. Matveev M. G., Mikhailov V. V., Sirota E. A. (2016) Combined prognostic model of a nonstationary multidimensional time series for constructing a spatial profile of atmospheric temperature. Vol. 22. No. 2. P. 89-94.

5. Xiaolei Xun, Jiguo Cao, Bani Mallick, Raymond J. Car-roll, Arnab Maity (2013) Parameter Estimation of Partial Differential Equation Models. Journal of the American Statistical Association. No 108:503. P. 1009-1020, DOI: $10.1080 / 01621459.2013 .794730$

6. Nosko V. P. (2002 ) Econometrica. Introduction to the regression analysis of time series. Moscow : NFPK. 273 p.

7. Gareth J., Witten D., Hastie Tr., Tibshirani R. (2013) An Introduction to Statisti-cal Learning. Springer. P. 40-48.
8. Matveev M. G., Kopytin A. V., Sirota E. A., Kopytina E. A. (2017) Modeling of Nonsta-tionary Distributed Processes on the Basis of Multidimensional Time Series. Procedia Engineering. - No. 201. P. 1-862. DOI: 10.1016/j. proeng. 2017.09. 643

9. Samarsky A. A. Theory of difference schemes. Moscow : Nauka, 1978. 376 p.

10. Matveev M. G., Sirota E. A. (2020) Analysis of the properties of the OLS-estimators in case of elimination of multi-collinearity in the problems of parametric identification of distributed dynamic processes. Bulletin of Voronezh state University, series "System analysis and information technologies". No 2. P. 15-22.

11. Matveev M. G., Sirota E. A. (2021) Analysis and investigation of the conservativeness condition in the problem of parametric identification of dynamic distributed processes. Journal of Physics: Conference Series, No 1902. 012079. DOI:10.1088/1742-6596/1902/1/012079

12. Kopytin A. V., Kopytina E. A., Matveev M. G. (2018) Application of the expanded Kalman filter for identifying the parameters of a distributed dynamical system. Vestnik VSU, series "System Analysis and Information Technologies". No. 3. P. 44-50.

13. Barseghyan A. A., Kupriyanov M. S., Kholod I. I., Tess M. D., Elizarov S. I. (2009) Analysis of data and processes: textbook. Manual. 3rd ed., reprint. and additional. St. Petersburg : BHV-Petersburg. $512 \mathrm{p}$.

14. Tibshirani R. J. (1996) Regression shrinkage and selection via the lasso. Journal of the Royal Statistical Society. Series B (Methodological). Vol. 58, No. 1. P. 267-288.

15. Ayvazyan S. A., Bukhstaber V. M., Enyukov I. S., Meshalkin L. D. (1989) Applied statistics. Classification and reduction of dimension. Moscow : Finance and Statistics. 607 p. 
Matveev Mikhail G. - DSc in Technical Sciences, Professor, Head of the Department of Information Technologies in Management, Faculty of Computer Sciences, Voronezh State University.

E-mail: mgmatveev@yandex.ru

ORCID iD: https://orcid.org/0000-0002-6528-6420

Sirota Ekaterina A. - PhD in Physics and Mathematics, Associate Professor, Department of Digital Technologies, Faculty of Computer Sciences.

E-mail: sirota_ea@sc.vsu.ru

ORCID iD: https://orcid.org/0000-0002-0338-7461 